\section{Oxalat(e) im Urin}

W. G. Guder

München, Deutschland

Synonym(e) Oxalsäure im Urin; Oxalurie

Englischer Begriff oxalates in urine; oxaluria; oxalic acid in urine

Definition Ausscheidung von Oxalsäure und ihren Salzen im Urin.

Funktion - Pathophysiologie Oxalat wird als Endprodukt des Glyoxylatstoffwechsels durch Sekretion im Tubulus ausgeschieden. Es wird neben enteraler Resorption durch Abbau von Glycin und Hydroxyprolin, aber auch von Ascorbinsäure im peroxisomalen Stoffwechsel gebildet. Pathophysiologisch ist es vor allem als Ursache für die Steinbildung in den ableitenden Harnwegen von Bedeutung.

Untersuchungsmaterial - Entnahmebedingungen Sammelurin, der mit $\mathrm{HCl}$ auf $\mathrm{pH}=$ ca. 1 gebracht wird $(5 \mathrm{~mL}$ 25 \%ige Salzsäure zum Sammelurin geben).

Probenstabilität Ohne Zusätze besonders bei neutralem und alkalischem $\mathrm{pH}$ weniger als 1 Stunde stabil. Im normalen angesäuerten Harn bis zu einem Tag stabil. Über längere Zeit nur bei Ansäuern stabil. Auch bei Kühlung und durch Einfrieren ist ohne Ansäuern keine Stabilität zu gewinnen. Daran ist auch die Ausfällung von Calciumoxalat beim Abkühlen und Einfrieren beteiligt, das beim Wiederauftauen nicht mehr in Lösung geht. Beim Mischen des Urins ist erst nach Zugabe der Säure zu zentrifugieren, um Verluste durch Sedimentbildung von Oxalatkristallen ( $\triangleright$ Oxalatkristalle) zu vermeiden.

\section{Analytik $>$ Oxalsäure.}

Konventionelle Einheit $\mathrm{mg} / 24 \mathrm{~h}$.

Internationale Einheit $\mathrm{mmol} / 24 \mathrm{~h}$.

Umrechnungsfaktor zw. konv. u. int. Einheit (mmol/ $24 \mathrm{~h}) \times 90=\mathrm{mg} / 24 \mathrm{~h}$.

Referenzbereich - Erwachsene $0,5 \mathrm{mmol} / 24 \mathrm{~h}(45 \mathrm{mg} /$ $24 \mathrm{~h})$.

Indikation Neben der seltenen Oxalose ist die Quantifizierung der Oxalate im Urin im Rahmen des SteinträgerMetaphylaxeprogramms indiziert ( $\triangleright$ Steinmetaphylaxe).

Diagnostische Wertigkeit Obwohl die Oxalatausscheidung nicht mit der Steinbildung direkt korreliert ist, wird seine erhöhte Ausscheidung als Ausdruck eines gesteigerten lithogenen Potenzials den steininhibierenden Faktoren Citrat ( $\vee$ Citrat im Urin), Magnesium und Harnvolumen gegenübergestellt und ist nur im Rahmen der Gesamtmusteranalyse zu interpretieren.

\section{Literatur}

Hesse A, Jahnen A, Klocke K, Nolde A, Scharrel O (1994) Nachsorge bei Harnsteinpatienten. Gustav Fischer-Verlag, Jena/Stuttgart 